\title{
Comparison of Transboundary Water Resources Allocation Models Based on Game Theory and Multi-Objective Optimization
}

\author{
Jisi Fu ${ }^{1}$, Ping-An Zhong ${ }^{1,2, *}$, Bin Xu ${ }^{1} \mathbb{C}$, Feilin Zhu ${ }^{1}$, Juan Chen ${ }^{1}$ and Jieyu Li ${ }^{1}$ \\ 1 College of Hydrology and Water Resources, Hohai University, No.1 Xikang Road, Nanjing 210098, China; \\ lcfujisi@163.com (J.F.); xubin_hhu@hhu.edu.cn (B.X.); zhufeilin@hhu.edu.cn (F.Z.); \\ chenjuanhhu@163.com (J.C.); hzlijy@163.com (J.L.) \\ 2 National Engineering Research Center of Water Resources Efficient Utilization and Engineering Safety, \\ Hohai University, No.1 Xikang Road, Nanjing 210098, China \\ * Correspondence: zpa_hhu@163.com or pazhong@hhu.edu.cn; Tel.: +86-135-0518-5185
}

check for updates

Citation: Fu, J.; Zhong, P.-A.; Xu, B.; Zhu, F.; Chen, J.; Li, J. Comparison of Transboundary Water Resources Allocation Models Based on Game Theory and Multi-Objective Optimization. Water 2021, 13, 1421. https://doi.org/10.3390/w13101421

Academic Editor: Francesco De Paola

Received: 18 April 2021

Accepted: 18 May 2021

Published: 20 May 2021

Publisher's Note: MDPI stays neutral with regard to jurisdictional claims in published maps and institutional affiliations.

Copyright: (c) 2021 by the authors. Licensee MDPI, Basel, Switzerland. This article is an open access article distributed under the terms and conditions of the Creative Commons Attribution (CC BY) license (https:// creativecommons.org/licenses/by/ $4.0 /)$.

\begin{abstract}
Transboundary water resources allocation is an effective measure to resolve water-related conflicts. Aiming at the problem of water conflicts, we constructed water resources allocation models based on game theory and multi-objective optimization, and revealed the differences between the two models. We compare the Pareto front solved by the AR-MOEA method and the NSGA-II method, and analyzed the difference between the Nash-Harsanyi Leader-Follower game model and the multiobjective optimization model. The Huaihe River basin was selected as a case study. The results show that: (1) The AR-MOEA method is better than the NSGA-II method in terms of the diversity metric $(\Delta)$; (2) the solution of the asymmetric Nash-Harsanyi Leader-Follower game model is a non-dominated solution, and the asymmetric game model can obtain the same water resources allocation scheme of the multi-objective optimal allocation model under a specific preference structure; (3) after the multi-objective optimization model obtains the Pareto front, it still needs to construct the preference information of the Pareto front for a second time to make the optimal solution of a multi-objective decision, while the game model can directly obtain the water resources allocation scheme at one time by participating in the negotiation. The results expand the solution method of water resources allocation models and provide support for rational water resources allocation.
\end{abstract}

Keywords: game theory; multi-objective optimization; water resources allocation; asymmetric NashHarsanyi Leader-Follower game model

\section{Introduction}

Transboundary water resources allocation usually involves multi-stakeholders. When water resources are insufficient to meet the water demand of the whole basin, water conflicts may arise among multi-stakeholders. In the case of water resources shortage, transboundary water resources allocation become an important means to solve water conflicts. Transboundary water resources allocation is a kind of complex decision-making problem with nonlinear, multi-objective and multi-stage characteristics.

In transboundary water resources allocation based on game theory, the way various stakeholders directly participate in the negotiation makes the water resources allocation results more acceptable. Roger [1] originally applied game theory to solve water resources conflicts. Since then, water resources allocation based on game theory has been studied. Bogardi and Szidarovsky [2] applied the oligopoly game to different water resources management problems. Parrachino et al. [3] applied cooperative game theory to solve water conflicts. Carraro et al. [4-6] systematically described the application of non-cooperative negotiation theory in water resources conflicts. Madani et al. [7] demonstrated that the application of water resources allocation based on game theory can be divided into five 
parts. Kerachian et al. [8] applied a fuzzy game to analyze the best strategy of various stakeholders. Wei et al. [9] applied a game theory-based model to analyze and solve water conflicts concerning water allocation and nitrogen reduction in the Middle Route of the South-to-North Water Transfer Project in China. Safari et al. [10] proposed a game model to analyze a water conflict in the Zarrinehrud River while considering agricultural demand and environmental constraints. Dinar et al. [11] summarized the application of game theory in water resources allocation. Degefu et al. [12] proposed a water allocation framework by combining the bankruptcy theory with the asymmetric Nash bargaining concept for solving the water sharing problem in transboundary river basins under scarcity. He et al. [13] presented a framework to allocate river water in a cooperative way based on game theory. Khachaturyan and Schoengold [14] applied game theory to solve the water shortage and pollution in the Kura-Araks basin. Yang et al. [15] proposed a repeated game model to analyze evolutionary transboundary cooperation.

The transboundary water resources allocation model based on multi-objective optimization focuses on solving water conflicts from the perspective of optimization objectives, and uses the multi-objective optimization method to achieve the water resources allocation results. In recent years, the multi-objective optimization algorithm has had the advantages of obtaining a complete Pareto front at one time, dealing with large-scale search space effectively and high universality, so the multi-objective optimization algorithm is widely used to the solve water resources allocation model based on multi-objective optimization. Rao et al. [16] applied a genetic algorithm to solve the water resources allocation model under drought conditions. Reed et al. [17] described the evolutionary multi-objective optimization in water resources. Tabari and Soltani [18] applied NSGA-II to solve the multiobjective optimal model for water resources allocation. Sepahvand et al. [19] developed the water resources allocation model based on bi-objective non-dominated sorting genetic algorithm-Type II. Qi et al. [20] constructed a multisource and multiuser water resources allocation model, and a genetic algorithm was used solve the model.

The above literature on transboundary water resources allocation seldom involve the comparative study of transboundary water resource allocation models based on game theory and multi-objective optimization. Moreover, from a mathematical point of view, the solution of multi-objective optimization is the closest solution to the ideal optimal point and the solution of game theory is the furthest distance to the worst solution. Therefore, it is necessary to make a comparative study of water resources allocation models based on game theory and multi-objective optimization. The asymmetric Nash-Harsanyi Leader-Follower game model proposed by Fu et al. comprehensively considers the principles of fairness, efficiency and sustainability, which can not only guarantee the basic water demand, but can also better balance the economic development levels among followers [21]. Therefore, this game model can obtain reasonable calculation results. The AR-MOEA method, which is applicable to a different front, is introduced to solve the transboundary water resources allocation model based on multi-objective optimization [22]. Therefore, this paper will explore the differences between the multi-objective optimization model based on the ARMOEA method and the asymmetric Nash-Harsanyi Leader-Follower game model in transboundary water resources allocation. First, we establish the transboundary water resources allocation model based on multi-objective optimization. Second, the AR-MOEA method is used to solve the Pareto front, and the Pareto front solved by AR-MOEA method is compared with the Pareto front solved by the widely used NSGA-II method. Finally, we analyze the relationship between the water resources allocation result of the NashHarsanyi Leader-Follower game model and Pareto front of multi-objective optimization solution, and illustrate the difference between water resources allocation based on the asymmetric Nash-Harsanyi Leader-Follower game model and water resources allocation based on the multi-objective optimization model.

The rest of this paper is organized as follows: Section 2 describes the material and methods. Section 3 describes the results of the asymmetric Nash-Harsanyi Leader-Follower game model and multi-objective optimization model based on the AR-MOEA method in the 
Huaihe River basin. Section 4 discusses the comparison of water resources allocation results based on the NSGA-II method and the AR-MOEA method, and reveals the differences between water resources allocation based on the asymmetric Nash-Harsanyi LeaderFollower game model and the multi-objective optimization model based on the AR-MOEA method. Section 5 concludes the paper.

\section{Materials and Methods}

\subsection{Study Area}

The Huaihe River basin located in the east (longitude from $111.9^{\circ}$ to $121.4^{\circ}$ ) and in the north (latitude from $30.9^{\circ}$ to $36.6^{\circ}$ ) of China, with a total area of $19,000 \mathrm{~km}^{2}$. The Huaihe River basin contains Henan province, Anhui province, and Jiangsu province (three provinces). The problem of water resources shortage is prominent, and the inter-provincial water resources conflict has become a serious problem. Though the study area is not international, an inter-provincial basin is effectively equivalent to an international basin as long as its boundaries do not match political boundaries. The stakeholders in the process of the Huaihe River basin water resources allocation are the watershed management agency, Henan province, Anhui province, and Jiangsu province. The spatial distribution and location of the associated province of Huaihe River basinis described in Figure 1.

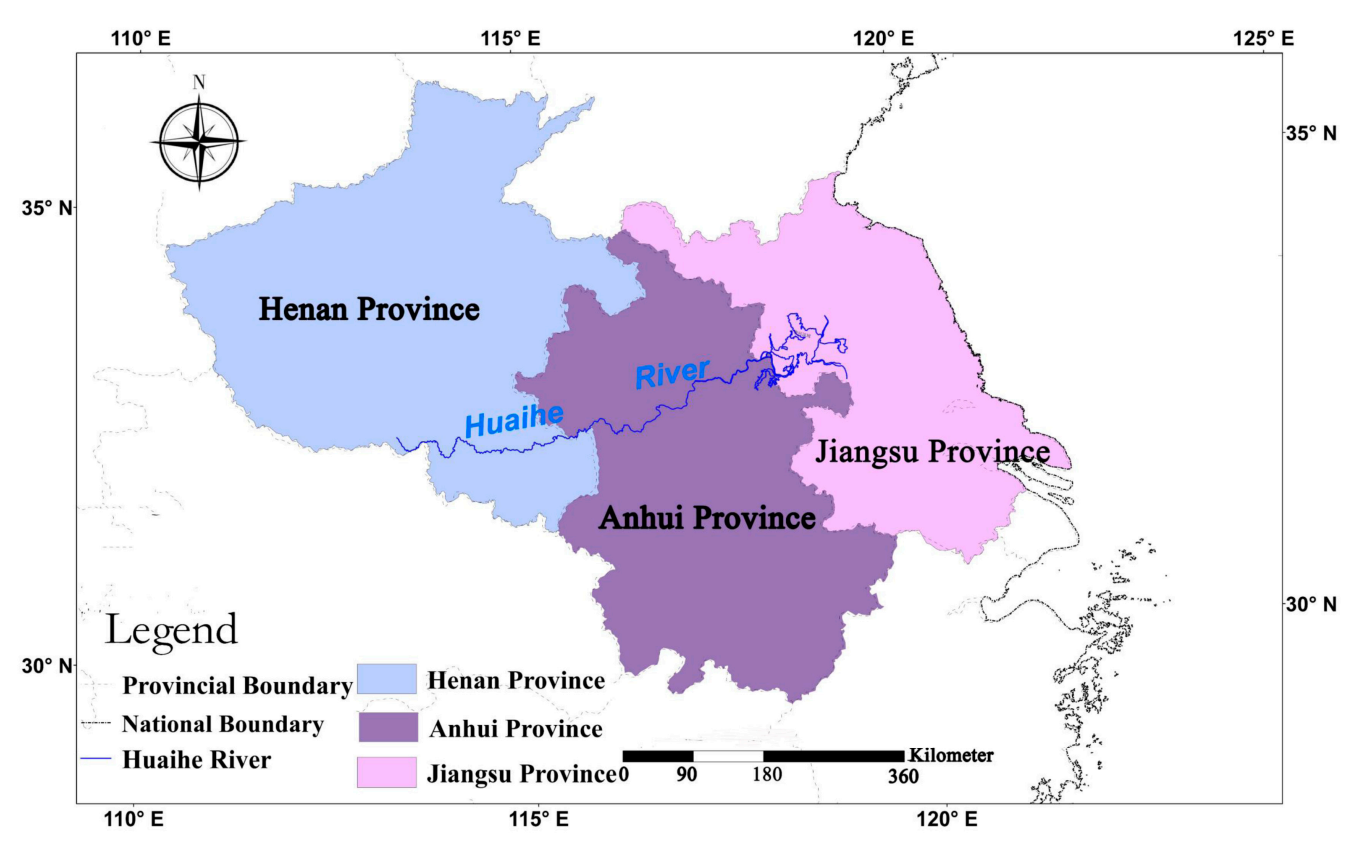

Figure 1. The spatial distribution and location of the associate province of Huaihe River basin.

\subsection{Transboundary Water Resources Allocation Based on Asymmetric Nash-Harsanyi Leader-Follower Game Model and Its Solution}

Transboundary water resources allocation based on the asymmetric Nash-Harsanyi Leader-Follower game model is proposed by Fu et al. [21]. The game model considering fairness, efficiency, and sustainable principles can not only guarantee the basic water demand, but also better balance the economic development levels among followers. Therefore, the game model is used to compare with the multi-objective optimization model.

Transboundary water resources allocation based on the asymmetric Nash-Harsanyi Leader-Follower game model is a combination of the leader-follower concepts and the Nash-Harsanyi theory. This model involves a single leader and multiple followers. In this study, a watershed management agency is considered to be the leader, who is responsible for the eco-environmental benefit of the whole basin. The associated areas serve as the followers. The eco-environmental water used by the whole basin in the allocation process is the public water. If the public water allocation is unreasonable, the eco-environmental 
situation will deteriorate, and sustainable development is challenged. Therefore, it is necessary to rationally distribute public water. The transboundary river water resources allocation process can be divided into two stages. The first stage involves allocating the public water. In the second stage, followers need to allocate water resources according to the asymmetric Nash-Harsanyi game model after the decisions are given by the watershed management agency.

The model of the watershed management agency (leader) can be described as the following:

$$
V=\max -\left(s-s^{*}\right)^{2},
$$

Subject to:

Water balance constraints:

$$
s+\sum_{i=1}^{n} w_{i}=Q,
$$

Public water constraints:

$$
0 \leq s \leq Q-\sum_{i=1}^{n} \lambda_{i}
$$

where $V$ is the objective function of the watershed management agency, $s$ is the public water and the solution of the leader, $s^{*}$ is the ideal eco-environmental water demand, $-\left(s-s^{*}\right)^{2}$ is the eco-environmental benefit, $w=\left\{w_{1}, w_{2}, \cdots, w_{n}\right\}$ is the decision vector of water allocated to followers, $w_{i}$ is the water in the $i$ th follower $(i=1,2, \cdots, n), Q$ is the total allocable water, and $\lambda_{i}$ is the minimum survival water demand in the $i$ th follower.

The model of the regions (followers) can be described as the following:

$$
\begin{gathered}
\max _{i=1}^{n}\left(u_{i}\left(w_{i}\right)-d_{i}\right)^{\alpha_{i},}, \\
d_{i}=u_{i}\left(I_{i}\right), \\
I_{i}=\max \left(\lambda_{i}, Q-\sum_{k \neq i} r_{i}-s\right), \\
\alpha_{i}=\eta \delta_{1 i}+(1-\eta) \delta_{2 i}, \\
\delta_{1 i}=\frac{r_{i}-I_{i}}{\sum_{i=1}^{n}\left(r_{i}-I_{i}\right)}, \\
\delta_{2 i}=\frac{\beta_{i}}{\sum_{i=1}^{n} \beta_{i}}, \\
\beta_{i}=1-\frac{D_{i}-\frac{1}{n} \sum_{i=1}^{n} D_{i}}{\frac{1}{n} \sum_{i=1}^{n} D_{i}}, \\
D_{i}=\sum_{k=1}^{e} \frac{r_{i, k}}{r_{i}} D_{i, k},
\end{gathered}
$$

Subject to:

Optimal solution existence constraints:

$$
u_{i}\left(w_{i}\right) \geq d_{i}, i=1,2, \cdots, n,
$$

Water balance constraints:

$$
s^{\prime}+\sum_{i=1}^{n} w_{i}=Q
$$


The bargaining weight constraints:

$$
\sum_{i=1}^{n} \alpha_{i}=1
$$

Water resources allocation constraints in each region:

$$
I_{i} \leq w_{i} \leq r_{i}
$$

where $u_{i}\left(w_{i}\right)$ is the objective function for follower $i, d_{i}$ is the disagreement point for follower $i, \alpha_{i}$ is the bargaining weight for follower $i, u_{i}\left(I_{i}\right)$ is the benefit of minimum water allocation for follower $i, r_{i}$ is the water demand for follower $i, I_{i}$ is the minimum water allocation for follower $i, \eta$ is the weight of the equity principle, $\delta_{1 i}$ is the bargaining weight for follower $i$ when only considering the equity principle that represents the equity of water for follower $i,(1-\eta)$ is the weight of efficiency principle, $\delta_{2 i}$ is the bargaining weight for follower $i$ when only the efficiency principle is considered, $\beta_{i}$ is the water use correction coefficient for follower $i, D_{i}$ is the integrated water utility for follower $i, r_{i, k}$ is the water demand of the $k$ th water user in the $i$ th follower, and $D_{i, k}$ is the integrated water utility of the $k$ th water user in the $i$ th follower.

The solution of the game model can refer to Fu's article [21]. After the watershed management agency obtains the public water, the followers can obtain the water resources allocation results by using the successive linear programming method.

The successive linear programming method was first proposed in 1961 [23]. It is an iterative optimization method using linear programming technology to solve nonlinear problems. At present, this method has been widely used in practical engineering and achieved good results. The steps for solving successive linear programming are as follows:

1. Let $x_{0}$ be the initial feasible solution of the optimization problem, and the corresponding objective function value is $f\left(x_{0}\right)$;

2. The nonlinear objective function is linearized by using the first order descriptive form point $\left(x_{0}, f\left(x_{0}\right)\right)$ of Taylor series, and the optimal solution $\left(x_{1}^{\prime}, f\left(x_{1}^{\prime}\right)\right)$ of linearized objective function in the neighborhood $\left(x_{0}-\delta, x_{0}-\delta\right)$ is obtained by using the linear programming method;

3. Taking the optimal solution $\left(x_{1}^{\prime}, f\left(x_{1}^{\prime}\right)\right)$ as a new feasible solution, the nonlinear objective function is linearized again by using the first order description point $\left(x_{1}^{\prime}, f\left(x_{1}^{\prime}\right)\right)$ of Taylor series, and the optimal solution $\left(x_{2}^{\prime}, f\left(x_{2}^{\prime}\right)\right)$ of linearized objective function in the neighborhood $\left(x_{1}-\delta, x_{1}-\delta\right)$ is obtained by using the linear programming method;

4. Repeat the above step 3 for the optimal solution and continue to iterate until the obtained optimal solution and the optimal objective function meet the condition of iteration termination.

\subsection{Transboundary Water Resources Allocation Based on Multi-Objective Optimization Model} and Its Solution

2.3.1. Transboundary Water Resources Allocation Based on Multi-Objective Optimization Model

Transboundary water resources allocation is a multi-stakeholders utilization conflict issue, involving watershed management agency and multiple areas.

The watershed management agency is responsible for the eco-environmental water utilization of the entire basin in the process of water resources allocation. The optimization goal of a watershed management agency is to maximize the eco-environmental benefit. The specific formula is as follows:

$$
F_{0}(s)=\max -\left(s-s^{*}\right)^{2}
$$


The optimization goal of area $i$ is to maximize the comprehensive benefit of the area. The specific calculation formula is as follows:

$$
F_{i}\left(w_{i}\right)=\max u_{i}\left(w_{i}\right)
$$

Constraints conditions for $n+1$ targets:

Water balance constraints:

$$
s^{\prime}+\sum_{i=1}^{n} w_{i}=Q
$$

Water resources allocation constraints in each region:

$$
I_{i} \leq w_{i} \leq r_{i}
$$

In the multi-objective optimization model of transboundary water resources allocation, the ecological environmental water used in the entire basin is public water. If the water resources allocation result is not reasonable, the eco-environmental situation is severe, and sustainable development is faced with challenges.

In the process of actual water resources allocation, due to the lack of consideration of eco-environmental water requirements, the eco-environmental water consumption is largely consumed by the production of water. This could not meet the sustainable development of water resources, which brings about water resources and eco-environmental problems, so the eco-environmental benefit of the watershed management agency needs to be prioritized. Therefore, the objective function of the watershed management agency in transboundary water resources allocation can be converted into constraint conditions, and the $n+1$ objective function converted into $n$ objective function.

\subsubsection{Multi-Objective Optimization Method}

(1) AR-MOEA method.

The AR-MOEA method is an evolutionary algorithm by Tian, which can be used to solve multi-objective optimization problems [22]. This method uses the enhanced inverted generational distance indicator as the main environmental selection strategy. In this method, a uniformly distributed group of reference points is used as the reference points set for calculating the enhanced inverted generational distance indicator. Therefore, the AR-MOEA method can be applied to a different front, and the distribution of reference points can be adjusted automatically according to the current population distribution in the calculation process.

The algorithm steps of AR-MOEA are as follows:

1. An initial population $P_{l}$ of size $M$ is randomly generated, save the population $P_{l}$ to an external document $E$, and multi-objective optimization method based on objective decomposition is used to generated a set of reference points $R$ with a scale of $M_{R}$;

2. A mating pool selection strategy based on the enhanced inverted generational distance with noncontributing solution detection is used to select the initial population $P_{l}$, mating pool $p_{l}^{\prime}$ is selected and the offspring population $P_{l}^{\prime}$ is generated;

3. The archive $E$ and the adapted reference point set $R^{\prime}$ are updated through the offspring population $P_{l}^{\prime}$, then the next generation population $P_{l+1}$ is selected by the enhanced inverted generational distance with a noncontributing solution detection selection strategy;

4. The above steps 1,2 , and 3 are repeated for the new initial population $P_{l+1}$ until a termination criterion is reached.

The AR-MOEA method contains four solutions sets: initial population $P_{l}$, initial reference point set $R$, archive set $E$, and adapted reference point set $R^{\prime}$. The population $P_{l}$ contains the candidate solutions as final output, the initial reference point set $R$ is used to guarantee uniform distribution of the candidate solution in $P_{l}$, and the archive set $E$ provides the shape of the Pareto front of the adapted reference point set $R^{\prime}$. An enhanced 
inverted generational distance indicator is used to select populations $P_{l}$ to change the adapted reference point set $R^{\prime}$. The relationships between the four solution sets are shown in Figure 2.

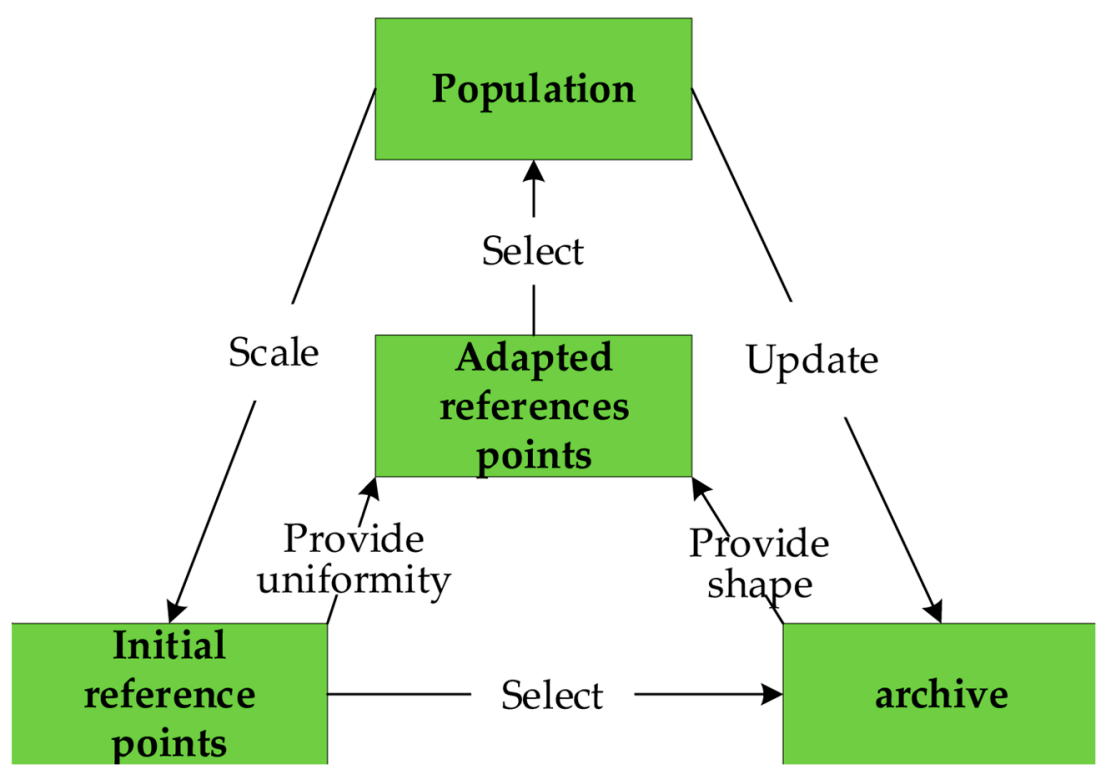

Figure 2. The relationship between the four solution sets.

(2) NSGA-II method.

The NSGA-II method is a multi-objective genetic algorithm proposed by Deb, which can be used to solve multi-objective optimization problems [24]. The NSGA-II method has the advantages of reducing the complexity of the algorithm, fast operation speeds, and good distribution of the solution set, so the method has become a widely used multi-objective genetic algorithm.

The algorithm steps of AR-MOEA are as follows:

1. An initial population $p_{l}$ of size $M$ is randomly generated, and the initial population is stratified by non-inferiority, and the genetic operator (selection operator, crossover operator, mutation operator) is used to obtain an offspring population $q_{l}$. Then, the initial population $p_{l}$ and the offspring population $q_{l}$ are mixed together to form a new population $N_{l}$ of size $2 M$;

2. The new population $N_{l}$ of size $2 M$ is rapidly sorted to form a non-dominated set $E_{l}$, then the crowding degree of the individuals in the non-dominated set $E_{l}$ is calculated. According to the non-dominant relationship and the crowding degree of individuals, a new initial population $p_{l+1}$ with the appropriate individual composition size of $M$ is selected;

3. The above steps 1 and 2 are repeated for the new initial population $p_{l+1}$ until a termination criterion is reached.

Figure 3 shows the specific calculation flow chart of NSGA-II.

(3) Linear weighted sum method.

The linear weighted sum method transforms the multi-objective optimization problem into a single objective problem to solve. The specific solving steps are as follows:

According to the importance of each goal, a set of non-negative weights $\tau_{i}(i=$ $1,2, \cdots, n)$ corresponding to the objective function $F_{i}$ is given, $\sum_{i=1}^{n} \tau_{i}=1$.The non-negative weight of the objective function is closely related to the preference structure information of the decision maker.

Each objective function is multiplied by its corresponding non-negative weight, then added to obtain a single objective function. 
The formula for the linear weighted sum method can be written as the following:

$$
\min F\left(w_{i}\right)=\sum_{i=1}^{n} \tau_{i} F_{i}\left(w_{i}\right), w_{i} \in W
$$

Subject to:

Inequality constraints:

$$
G_{i}\left(w_{i}\right) \geq 0
$$

Equality constraints:

$$
H_{i}\left(w_{i}\right)=0
$$

The linear weighted sum method can be solved by the successive linear programming method.

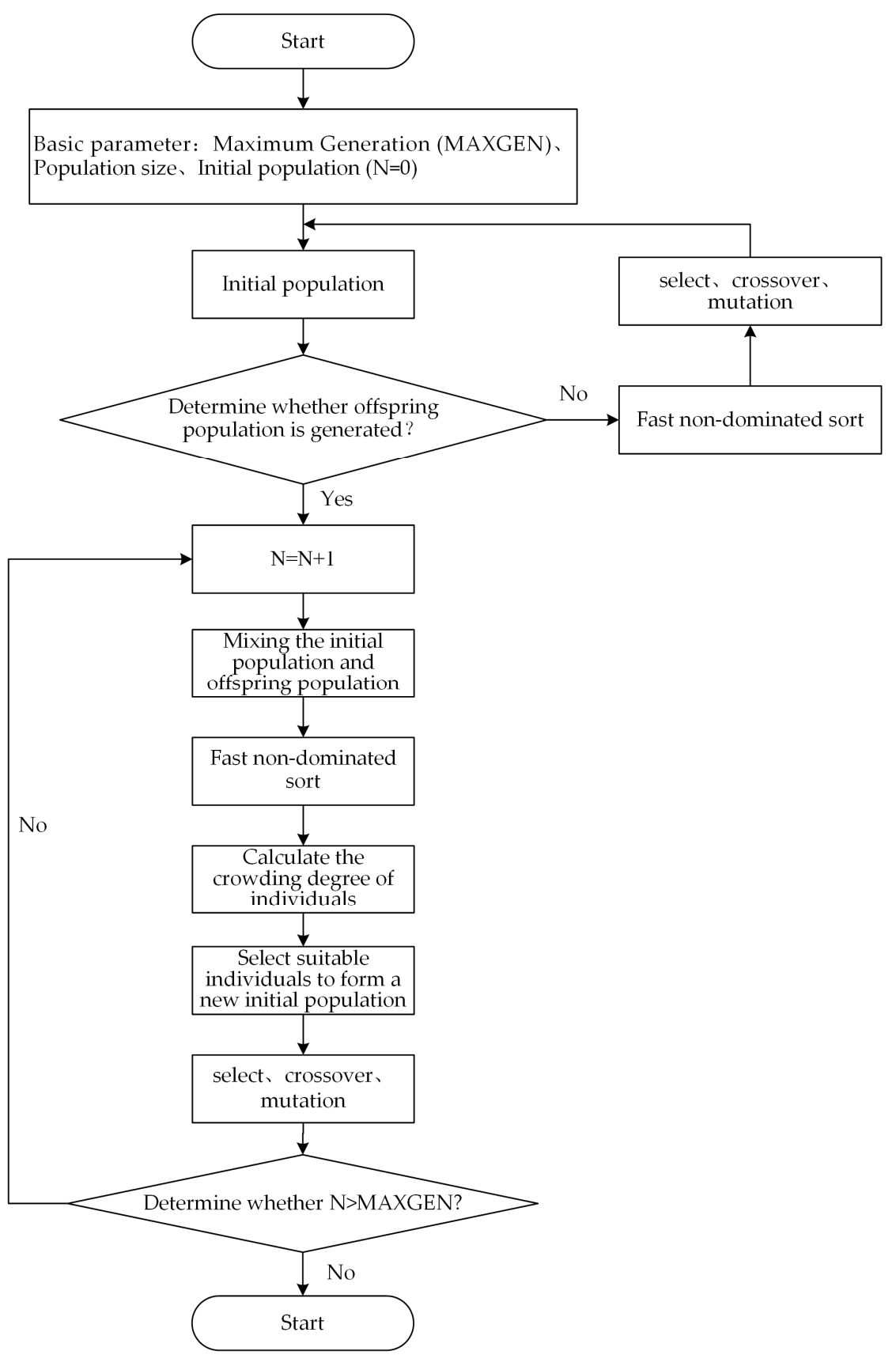

Figure 3. Calculation flow chart of NSGA-II. 


\subsubsection{Performance Metrics for Multi-Objective Optimization Evolutionary Algorithms}

According to the characteristics of the solution set measured by the performance metrics, the existing algorithm performance metrics can be divided into three categories:

(1) Convergence metrics - evaluate the approximation degree between the true Pareto front and non-dominated solution.

Generational distance (GD) can be used to measure the convergence of non-dominated solutions obtained by multi-objective evolutionary algorithms [25]. The smaller the GD is, the better the convergence of non-dominated solutions obtained by multi-objective evolutionary algorithms is. It is assumed that $P^{*}$ is a set of solutions uniformly distributed on the real Pareto front and $A$ is the non-dominated solution set obtained by multi-objective evolutionary algorithms. The expression of GD can be defined as:

$$
\operatorname{GD}\left(A, P^{*}\right)=\frac{\sqrt{\sum_{y \in A} \min _{x \in P^{*}}(x, y)^{2}}}{|A|},
$$

where $d(x, y)$ is the Euclidean distance between point $x$ in $P^{*}$ and point $y$ in $A$, and $|A|$ is the number of non-dominated solutions.

(2) Diversity metrics - evaluate scatter of non-dominated solutions. Diversity metrics can be subdivided into the evenness and spread of the non-dominated solutions in the whole feasible region. Evenness in diversity metrics reflects the evenness of distribution in non-dominated solutions. Spread in diversity metrics reflects the spread degree of solution set in target space.

A range metric $(\Delta)$ is employed to measure the diversity of non-dominated solutions obtained by the multi-objective evolutionary algorithm [24]. The mathematical definition of diversity metrics is described as:

$$
\Delta=\frac{d_{f}+d_{l}+\sum_{i=1}^{N-1}\left|d_{i}-\bar{d}\right|}{d_{f}+d_{l}+(N-1) \bar{d}},
$$

where $d_{f}$ and $d_{l}$ are the Euclidean distance between the extreme non-dominated solution and the boundary solutions of the obtained non-dominated solution set, $N$ is the number of non-dominated solutions obtained by multi-objective evolutionary algorithms, $d_{i}$ is the distance between continuous solutions in obtained non-dominated solution set, and $\bar{d}$ is the average of all $d_{i}(i=1,2, \ldots, N-1)$. When $\Delta$ is zero, it indicates that the non-dominated solution set calculated by the multi-objective optimization algorithm has good diversity.

(3) Composite metrics considering convergence and diversity.

Inverted generational distance (IGD) and hypervolume (HV) are employed to measure the convergence and diversity of a non-dominated solution obtained by the multi-objective evolutionary algorithm.

IGD can be used to measure the mean of the minimum distance between point $x$ in $P^{*}$ and point $y$ in $A$. The smaller IGD is, the better the convergence and diversity of non-dominated solutions obtained by multi-objective evolutionary algorithms is. The expression of IGD can be described as the following:

$$
\operatorname{IGD}\left(A, P^{*}\right)=\frac{\sum_{x \in P^{*}} \min _{y \in A} d(x, y)}{\left|P^{*}\right|},
$$

where $d(x, y)$ is the Euclidean distance between point $x$ in $P^{*}$ and point $y$ in $A,\left|P^{*}\right|$ is the number of true Pareto solutions.

$\mathrm{HV}$ is the volume in the target space region enclosed by the non-dominated solutions $A$ and the reference point set $R$ [25]. The larger the HV, the better the convergence and 
diversity of non-dominated solutions obtained by multi-objective evolutionary algorithms. The calculation formula of $\mathrm{HV}$ is:

$$
\mathrm{HV}=\operatorname{volume}\left(\cup_{i=1}^{N} v_{i}\right),
$$

where volume $(\cdot)$ is Lebesgue measure, $N$ is the number of non-dominated solutions obtained by multi-objective evolutionary algorithms, and $v_{i}$ is the volume enclosed between the $i$ th non-dominated solution and the reference point.

We selected the above representative performance metrics to evaluate the AR-MOEA method and the NSGA-II method.

\subsection{Data of Nash-Harsanyi Leader-Follower Game Model and Multi-Objective Optimization Model}

The stakeholders in the Huaihe River basin are the watershed management agency and the three previously mentioned provinces. According to the two water resources allocation models mentioned in Sections 2.2 and 2.3, the data required for the two models are total allocable water $Q$, ideal eco-environmental water demand $s^{*}$, weight of the equity principle $\eta$, weight of efficiency principle $(1-\eta)$, water demand in each province $r_{i}$, minimum survival water demand in each province $\lambda_{i}$, minimum water allocation in each province $I_{i}$, disagreement point in each province $d_{i}$, bargaining weight in each province $\alpha_{i}$, objective function in each province $u_{i}\left(w_{i}\right)$, and integrated water utility in each province $D_{i}$. The data used in the two models are all taken from Fu et al. (2018) [21]. Table 1 shows the data required for two transboundary water resources allocation models.

Table 1. Data required for two transboundary water resources allocation models.

\begin{tabular}{|c|c|c|c|}
\hline Parameter & Henan Province & Anhui Province & Jiangsu Province \\
\hline$Q$ & & 400.5 (Hundred million $\mathrm{m}^{3}$ ) & \\
\hline$s^{*}$ & & 100.1 (Hundred million $\mathrm{m}^{3}$ ) & \\
\hline$\eta$ & & 0.667 & \\
\hline$(1-\eta)$ & & 0.333 & \\
\hline$r_{i}$ & 126.4 (Hundred million $\mathrm{m}^{3}$ ) & 135.2 (Hundred million $\mathrm{m}^{3}$ ) & 137.3 (Hundred million $\mathrm{m}^{3}$ ) \\
\hline$\lambda_{i}$ & 27.7 (Hundred million $\mathrm{m}^{3}$ ) & 37.0 (Hundred million $\mathrm{m}^{3}$ ) & 50.4 (Hundred million $\mathrm{m}^{3}$ ) \\
\hline$I_{i}$ & 27.9 (Hundred million $\mathrm{m}^{3}$ ) & 37.0 (Hundred million $\mathrm{m}^{3}$ ) & 50.4 (Hundred million $\mathrm{m}^{3}$ ) \\
\hline$d_{i}$ & 273.443 (Hundred million yuan) & 289.476 (Hundred million yuan) & 389.663 (Hundred million yuan) \\
\hline$D_{i}$ & $666.8\left(\mathrm{~m}^{3} /\right.$ Hundred million yuan) & $1035.2\left(\mathrm{~m}^{3} /\right.$ Hundred million yuan) & $1034.7\left(\mathrm{~m}^{3} /\right.$ Hundred million yuan) \\
\hline$\alpha_{i}$ & 0.423 & 0.288 & 0.289 \\
\hline$u_{i}\left(w_{i}\right)$ & $\begin{array}{c}u_{1}\left(w_{1}\right)=-0.0302 \widetilde{w}_{1}^{2}+10.5478 \widetilde{w}_{1} \\
+2.6678\end{array}$ & $\begin{array}{c}u_{2}\left(w_{2}\right)=- \\
+0.0254 \widetilde{w}_{2}^{2}+8.6364 \widetilde{w}_{2} \\
+0.1733\end{array}$ & $\begin{array}{c}u_{3}\left(w_{3}\right)=-0.024 \widetilde{w}_{3}^{2}+8.9395 \widetilde{w}_{3} \\
+0.0763\end{array}$ \\
\hline
\end{tabular}

\section{Results}

3.1. Water Resources Allocation Based on Asymmetric Nash-Harsanyi Leader-Follower Game Model and Its Solution in Huaihe River Basin

For the four stakeholders in the Huaihe River basin, the watershed management agency serves as leader and the three provinces serve as followers. The model of the watershed management agency is as follows:

$$
V=\max -(s-100.5)^{2}
$$

Subject to:

Water balance constraints:

$$
s+\sum_{i=1}^{n} w_{i}=400.5
$$

Public water constraints:

$$
0 \leq s \leq 285.4
$$

The public water $s$ obtained by the above model of watershed management agency is 100.1 hundred million $\mathrm{m}^{3}$. 
After the watershed management agency determines that the public water to be 100.1 hundred million $\mathrm{m}^{3}$, the model of the three provinces is as follows:

$$
\begin{aligned}
\max \left(u_{1}-d_{1}\right)^{\alpha_{1}}\left(u_{2}-d_{2}\right)^{\alpha_{2}}\left(u_{3}-d_{3}\right)^{\alpha_{3}} \\
=\max \left(-0.0302 w_{1}^{2}+10.5478 w_{1}+2.6678-273.443\right)^{0.373} \\
\quad\left(-0.0254 w_{2}^{2}+8.6364 w_{2}+0.1733-289.476\right)^{0.323}, \\
\quad\left(-0.024 w_{3}^{2}+8.9395 w_{3}+0.0763-389.663\right)^{0.300}
\end{aligned}
$$

Subject to:

Optimal solution existence constraints:

$$
\begin{aligned}
& u_{1} \geq 273.443, \\
& u_{2} \geq 289.476, \\
& u_{3} \geq 389.663
\end{aligned}
$$

Water balance constraints:

$$
w_{1}+w_{2}+w_{3}+100.1=400.5
$$

The water allocation constraints in Henan province:

$$
27.9 \leq w_{1} \leq 126.4
$$

The water allocation constraints in Anhui province:

$$
37.0 \leq w_{2} \leq 135.2
$$

The water allocation constraints in Jiangsu province:

$$
50.4 \leq w_{3} \leq 137.3
$$

We used the successive linear programming method to solve the above model for the three provinces. The amount of water allocated to Henan province, Anhui province, and Jiangsu province were 95.5 hundred million $\mathrm{m}^{3}, 97.5$ hundred million $\mathrm{m}^{3}$, and 107.4 hundred million $\mathrm{m}^{3}$, respectively, and the satisfaction of rates in the corresponding provinces were $68.6 \%, 61.6 \%$, and $65.6 \%$, respectively. In addition, the benefit of the three provinces were 734.6 hundred billion yuan, 600.8 hundred million yuan, and 683.3 hundred million yuan; the total benefit was 2018.7 hundred million yuan.

\subsection{Water Resources Allocation Based on Multi-Objective Optimization Model and Its Solution in} Huaihe River Basin

In the water resources allocation based on multi-objective optimization model, the eco-environmental benefit $F_{0}$ of the watershed management agency is given priority, so the public water allocated to watershed management agency is 100.1 hundred million $\mathrm{m}^{3}$. The objective function of the watershed management agency in transboundary water resources allocation can be converted into constraint conditions, then four objective functions converted into three objective functions. Water resources allocation based on the multi-objective optimization model is established as follows:

The objective function of Henan province is:

$$
F_{1}=\max \left(-0.0302 w_{1}^{2}+10.5478 w_{1}+2.6678\right)
$$

The objective function of Anhui province is:

$$
F_{2}=\max \left(-0.0254 w_{2}^{2}+8.6364 w_{2}+0.1733\right)
$$


The objective function of Jiangsu province is:

$$
F_{3}=\max \left(-0.024 w_{3}^{2}+8.9395 w_{3}+0.0763\right)
$$

Subject to:

Water balance constraints:

$$
w_{1}+w_{2}+w_{3}=400.5-100.1
$$

The water allocation constraints in Henan province:

$$
27.9 \leq w_{1} \leq 126.4
$$

The water allocation constraints in Anhui province:

$$
37.0 \leq w_{2} \leq 135.2
$$

The water allocation constraints in Jiangsu province:

$$
50.4 \leq w_{3} \leq 137.3,
$$

where $w_{1}$ is the amount of water allocated to Henan province, $w_{2}$ is the amount of water allocated to Anhui province, and $w_{3}$ is the amount of water allocated to Jiangsu province.

The NSGA-II method and the AR-MOEA method are used to solve the multi-objective optimization model established above. The population size of the two methods (NSGA-II method and AR-MOEA method) are both 100, and the maximum iterations of the two methods are both 1000. The multi-objective optimization model based on the NSGA-II method and the AR-MOEA method are both solved by using PlatEMO platform [26].

The Pareto front of multi-objective optimization based on NSGA-II method in the Huaihe River basin is shown in Figure 4. As can be seen from Figure 4, the points on the Pareto front solved by NSGA-II method are not uniformly distributed.

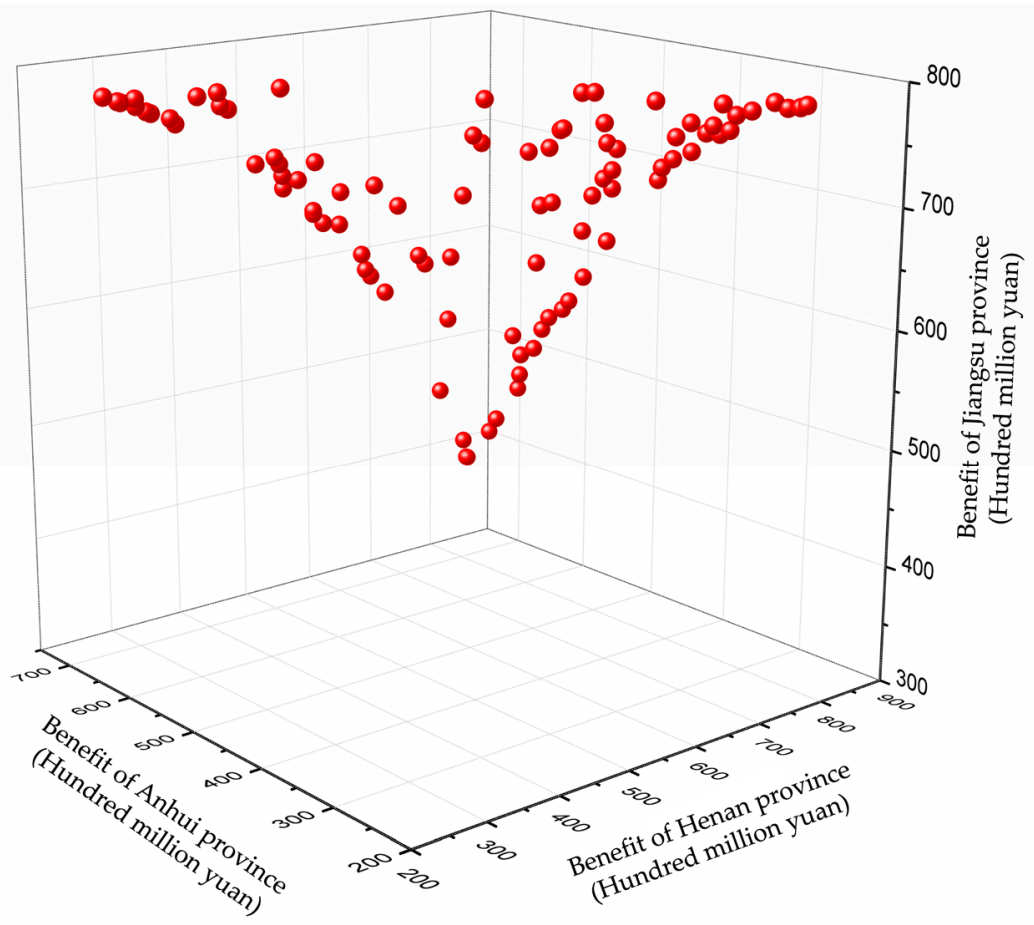

Figure 4. Pareto front of multi-objective optimization based on the NSGA-II method in the Huaihe River basin. 
Figure 5 shows the Pareto front of multi-objective optimization based on the ARMOEA method in the Huaihe River basin. In Figure 5, the points on the Pareto front solved by the AR-MOEA method are uniformly distributed.

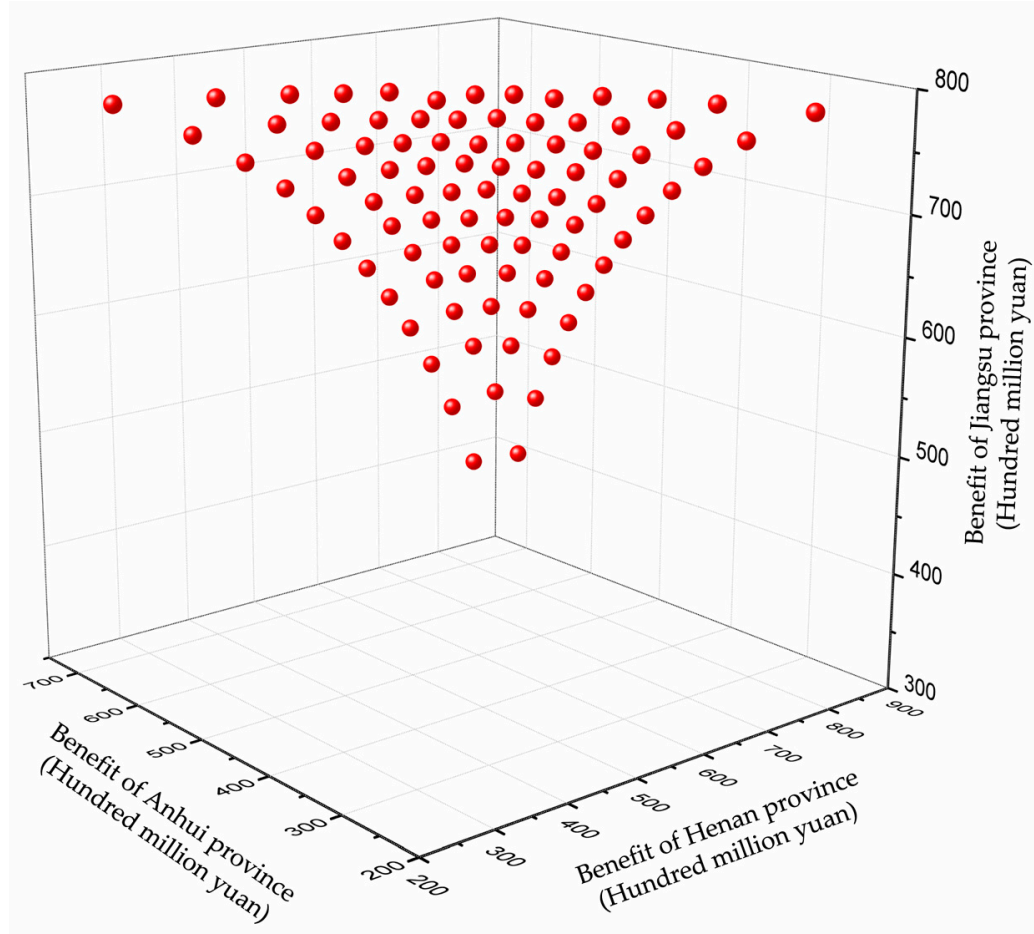

Figure 5. Pareto front of multi-objective optimization based on the AR-MOEA method in the Huaihe River basin.

\section{Discussion}

4.1. Comparison of Water Resource Allocation Results Based on NSGA-II Method and AR-MOEA Method

In order to measure the effect of the NSGA-II method and the AR-MOEA method on multi-objective optimization in the three provinces of the Huaihe River basin, we introduced three categories of performance metrics (convergence metrics, diversity metrics, and the metrics considering convergence and diversity), as mentioned in Section 2.3.3. The average value and significance test of algorithm performance metrics of the two methods running for 30 times are shown in Table 2.

Table 2. The average value and significance test of algorithm performance metrics of the two methods running for 30 times.

\begin{tabular}{cccc}
\hline \multirow{2}{*}{ Metrics } & \multicolumn{2}{c}{ Average Value } & \multirow{2}{*}{ Significance Test } \\
\cline { 2 - 3 } & NSGA-II Method & AR-MOEA Method & \\
\hline Convergence metric & 17.18 & 16.99 & $\mathrm{~h}=0^{1}$ \\
GD & 2.74 & 1.97 & $\mathrm{~h}=1^{2}$ \\
Diversity metric $\Delta$ & 40.94 & 40.93 & $\mathrm{~h}=0$ \\
Composite metric & 3.22 & 3.30 & $\mathrm{~h}=0$ \\
IGD & Composite metric HV & & \\
\hline
\end{tabular}

${ }^{1}$ indicates that significance test does not reject the null hypothesis at the $5 \%$ significance level, this means that there is no significant difference in the values of algorithm performance metrics of two methods; ${ }^{2}$ indicates that significance test rejects the null hypothesis at the $5 \%$ significance level, this means that there is significant difference in the values of algorithm performance metrics of two methods. 
Table 2 shows:

1. The convergence metric (GD) and composite metrics (IGD, HV) pass a $5 \%$ significance level; this means that there is no significant difference in convergence metric (GD) and composite metrics (IGD, HV). Therefore, we think that the two methods have almost the same effect on convergence metric (GD) and composite metrics (IGD, HV);

2. The diversity metric $(\Delta)$ cannot pass a $5 \%$ significance level; this means that there is a significant difference in the diversity metric $(\Delta)$. As for the diversity metric $(\Delta)$, when $\Delta$ tends to zero, it indicates that the non-dominated solution set calculated by the multi-objective optimization algorithm has good diversity. The average value of the diversity metric $(\Delta)$ in the AR-MOEA method is lower than that in the NSGA-II method. Therefore, the AR-MOEA method is better than the NSGA-II method in terms of the diversity metric $(\Delta)$.

In conclusion, the AR-MOEA method is better than the NSGA-II method in terms of the diversity metric $(\Delta)$.

\subsection{Comparison of Water Resource Allocation Results Based on Multi-Objective Optimization Model and Game Model}

Because the AR-MOEA method is superior in the diversity metric $(\Delta)$ when solving the multi-objective optimization model of the Huaihe River basin water resources allocation, we choose the AR-MOEA method to solve the multi-objective optimization model of water resource allocation in the Huaihe River basin. Then, we compared the water resources allocation results based on the the asymmetric Nash-Harsanyi Leader-Follower game model and the multi-objective optimization model based on the AR-MOEA method. The benefits for the three provinces of Huaihe River obtained by the two model are shown in Figure 6.

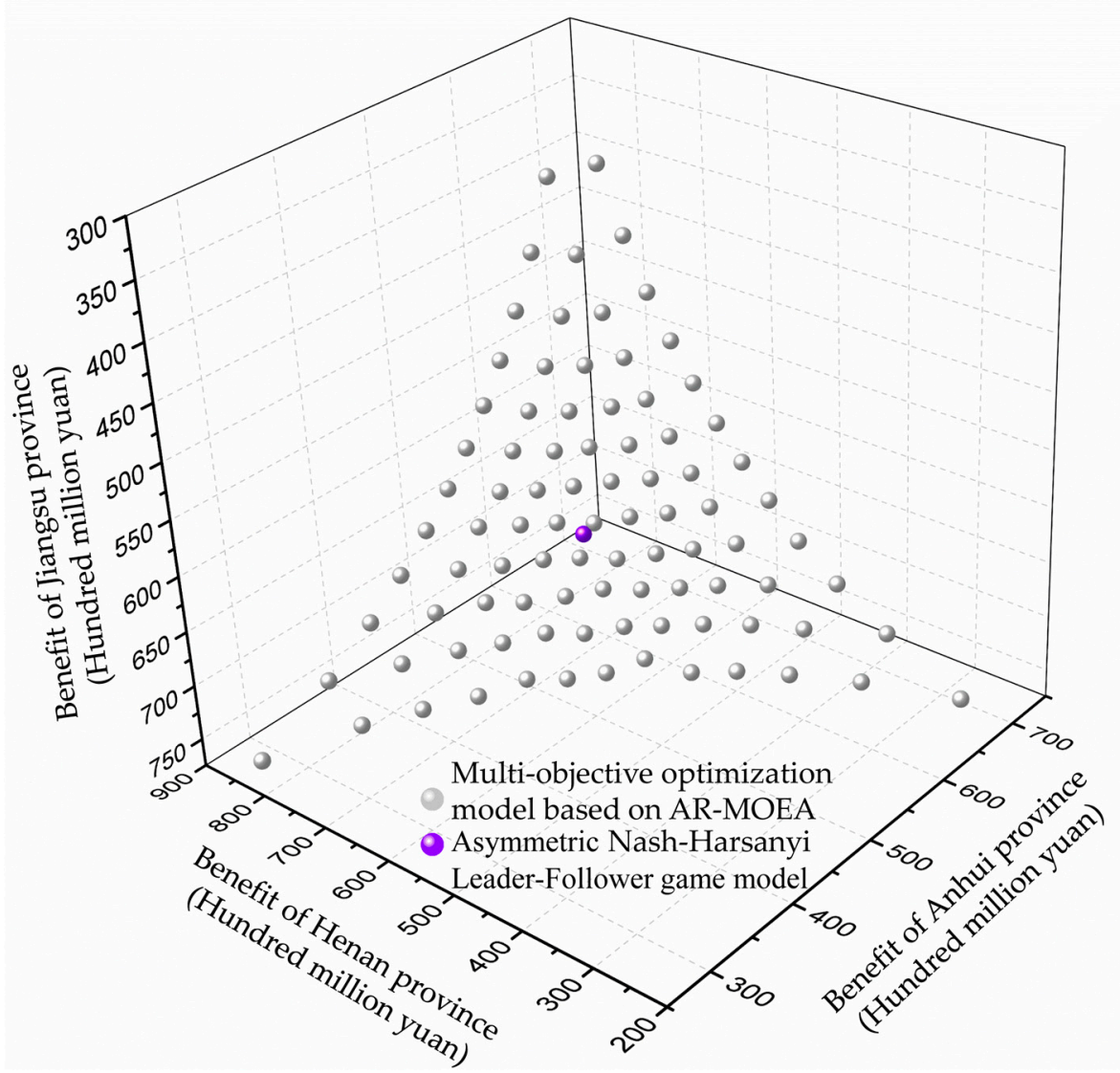

Figure 6. The benefits of three provinces of the Huaihe River obtained by the two models. 
Figure 6 shows that the water resources allocation result of the asymmetric NashHarsanyi Leader-Follower game model is on the Pareto front solved by the AR-MOEA method, that is, the solution of the asymmetric Nash-Harsanyi Leader-Follower game model is a non-dominated solution.

The multi-objective optimization solution is the closest solution to the ideal optimal point, and the ideal optimal point is different under different preference information. The asymmetric Nash-Harsanyi Leader-Follower game model considers the principles of fairness and efficiency, so the solution nearest to the ideal optimal point under the principle of efficiency and the principle of fairness is chosen for the multi-objective optimization solution. The multi-objective optimization solution under the principle of efficiency can be converted into solving non-dominated solution of maximum comprehensive benefit. The non-dominated solution with the maximum comprehensive benefit can be obtained by the linear weighted sum method with equal non-negative weights. The multi-objective optimization solution under the principle of fairness can be converted into solving nondominated solution of equal water demand satisfaction rate. The non-dominated solution with an equal water demand satisfaction rate can be solved by the water balance equation and an equal water demand equation in the three provinces of the Huaihe River basin.

The water resources allocation results in the three provinces of the Huaihe River basin solved by the multi-objective optimization solution under different preference information (focusing on overall comprehensive benefit or overall fairness) and the asymmetric NashHarsanyi Leader-Follower game model (considering the principle of efficiency and fairness) were compared and analyzed, as shown in Table 3.

Table 3. Water resources allocation results under different scenarios.

\begin{tabular}{|c|c|c|c|c|}
\hline \multirow{2}{*}{ Province } & \multirow{2}{*}{ Parameters } & \multicolumn{2}{|c|}{ Multi-Objective Optimization Solution } & \multirow{2}{*}{$\begin{array}{l}\text { Asymmetric Nash-Harsanyi } \\
\text { Leader-Follower Game Mode }\end{array}$} \\
\hline & & Efficiency & Fairness & \\
\hline \multirow{3}{*}{ Henan } & $w_{1}\left(\right.$ Hundred million $\left.\mathrm{m}^{3}\right)$ & 107.8 & 92.2 & 95.5 \\
\hline & $F_{1}$ (Hundred million yuan) & 788.8 & 718.4 & 734.6 \\
\hline & water demand satisfaction rate (\%) & 81.1 & 65.3 & 68.6 \\
\hline \multirow{3}{*}{ Anhui } & $w_{2}\left(\right.$ Hundred million $\left.\mathrm{m}^{3}\right)$ & 90.5 & 101.1 & 97.5 \\
\hline & $F_{2}$ (Hundred million yuan) & 573.7 & 613.7 & 600.8 \\
\hline & water demand satisfaction rate $(\%)$ & 54.5 & 65.3 & 61.6 \\
\hline \multirow{3}{*}{ Jiangsu } & $w_{3}\left(\right.$ Hundred million $\mathrm{m}^{3}$ ) & 102.1 & 107.1 & 107.4 \\
\hline & $F_{3}$ (Hundred million yuan) & 662.6 & 682.2 & 683.3 \\
\hline & water demand satisfaction rate $(\%)$ & 59.5 & 65.3 & 65.6 \\
\hline \multicolumn{2}{|c|}{ Total benefit (Hundred million yuan) } & 2025.1 & 2014.3 & 2018.7 \\
\hline
\end{tabular}

Table 3 shows:

1. The water demand satisfaction rate of the three provinces of the Huaihe River basin is not balanced in the multi-objective optimization solution under the principle of efficiency, so the fairness of water resources allocation result in the multi-objective optimization solution under the principle of efficiency is ignored. The water demand satisfaction rate of Henan province is higher than that of Anhui province and Jiangsu province, which makes Anhui province and Jiangsu province unable to accept the water resources allocation scheme;

2. The multi-objective optimization solution under the principle of fairness satisfies the fairness of water resources allocation result in the three provinces, but the total benefit of the multi-objective optimization solution under the principle of fairness is lower than the water resources allocation result solved by the asymmetric Nash-Harsanyi Leader-Follower game model. The above analysis makes the three provinces unable to accept the water resources allocation scheme;

3. The water resources allocation result of the asymmetric Nash-Harsanyi LeaderFollower game model is a compromise solution between two kinds of multi-objective optimization solutions under different preference information (focusing on overall 
comprehensive benefit or overall fairness). The efficiency and fairness are considered comprehensively in the game model, which makes it easier for the three provinces to accept the water resources allocation scheme.

In order to explore the relationship between the water resources allocation results obtained by the linear weighted sum method with different objective function non-negative weights and the water resource allocation results based on the asymmetric Nash-Harsanyi Leader-Follower game model, the non-negative weights of objective function in linear weighted sum method are fitted by using a trial and error method.

When the non-negative weights of Henan province, Anhui province, and Jiangsu province are $0.281,0.364$, and 0.355 , respectively, the water resources allocation obtained by the linear weighted sum method are equal to the water resources allocation results obtained by the asymmetric Nash-Harsanyi Leader-Follower game model. This result shows that the multi-objective optimization model can obtain the same water resources scheme as the Nash-Harsanyi Leader-Follower game model under a specific preference structure.

After the multi-objective optimization model obtains the Pareto front, it still needs to construct the preference information of Pareto front for the second time to make the optimal coordinated solution of the multi-objective decision. In this kind of integrated water resources allocation, each stakeholder cannot directly participate in water resources allocation, and the behavior influence of various stakeholders cannot be taken into account, which may make the obtained water resources unacceptable to various stakeholders. The asymmetric Nash-Harsanyi Leader-Follower game model can not only guarantee basic water demand, but can also better balance the economic development level among the three provinces. Moreover, in the asymmetric Nash-Harsanyi Leader-Follower game model, the three provinces of the Huaihe River basin can directly participate in the negotiation to make the water resources allocation results acceptable to all stakeholders, and the obtained result has a practical guiding role in water resources allocation.

\section{Conclusions}

In this study, we analyzed the difference between the asymmetric Nash-Harsanyi Leader-Follower game model and the multi-objective optimization model in water resources allocation. First, we established a transboundary water resources allocation model based on multi-objective optimization. Second, the AR-MOEA method was used to solve the Pareto front, and the Pareto front solved by AR-MOEA method was compared with the Pareto front solved by NSGA-II. Finally, we analyzedthe relationship between the water resources allocation result of the Nash-Harsanyi Leader-Follower game model and the Pareto front of the multi-objective optimization solution, and illustrated the difference between the asymmetric Nash-Harsanyi Leader-Follower game model and the multi-objective optimization model in water resources allocation. The asymmetric NashHarsanyi Leader-Follower game model and the multi-objective optimization model based on AR-MOEA method were applied to the Huaihe River basin. The main conclusions are as follows:

1. The AR-MOEA method and the NSGA-II method were applied to solve the multiobjective optimization model of the Huaihe River basin water resources allocation. The results show that the AR-MOEA method is better than the NSGA-II method in terms of the diversity metric $(\Delta)$;

2. The solution of the asymmetric Nash-Harsanyi Leader-Follower game model is a non-dominated solution, and the asymmetric Nash-Harsanyi Leader-Follower game model can obtain the same water resources allocation scheme of the multi-objective optimal allocation model under a specific preference structure;

3. After the multi-objective optimization model obtains the Pareto front, it still needs to construct the preference information of the Pareto front for the second time to make the optimal solution of multi-objective decision, while the game model can directly obtain the water allocation scheme in one time by participating in the negotiation. 
Author Contributions: Conceptualization, J.F. and P.-A.Z.; methodology, J.F. and B.X.; data curation, J.F. and J.L.; writing—original draft preparation, J.F.; writing—review and editing, J.C. and F.Z. All authors have read and agreed to the published version of the manuscript.

Funding: This research was funded by the Fundamental Research Funds for the Central Universities (grant numbers 2018B606X14, B200202028, B200201030, B200202032, B200204038), the Postgraduate Research \& Practice Innovation Program of Jiangsu Province (grant number KYCX18_0578), the National Natural Science Foundation of China (grant numbers 52079037, 52009029, 51909062), the National Key R\&D Program of China (grant number 2017YFC0405606), and the China Postdoctoral Science Foundation Funded Project (grant number 2018T110525).

Institutional Review Board Statement: Not applicable.

Informed Consent Statement: Not applicable.

Data Availability Statement: All data generated or analyzed during this study are included in this published article.

Acknowledgments: We thank the anonymous reviewers for their constructive reviews and the editor and associate editor for their remarks. We wish to thank Lucy Marshall (Water Research Centre, School of Civil and Environmental Engineering, UNSW Sydney) for her support during the research activities.

Conflicts of Interest: The authors declare no conflict of interest.

\section{References}

1. Rogers, P. A game theory approach to the problems of international river basins. Water Resour. Res. 1969, 5, 749-760. [CrossRef]

2. Bogardi, I.; Szidarovsky, F. Application of game theory in water management. Appl. Math. Model. 1976, 1, 16-20. [CrossRef]

3. Parrachino, I. Cooperative Game Theory and Its Application to Natural, Environmental and Water Resource Issues; World Bank Publications: Washington, DC, USA, 2005.

4. Carraro, C.; Marchiori, C.; Sgobbi, A. Applications of Negotiation Theory to Water Issues; World Bank Publications: Washington, DC, USA, 2005.

5. Carraro, C.; Marchiori, C.; Sgobbi, A. Negotiating on water: Insights from non-cooperative bargaining theory. Environ. Dev. Econ. 2007, 12, 329-349. [CrossRef]

6. Carraro, C.; Sgobbi, A. Modelling negotiated decision making in environmental and natural resource management: A multilateral, multiple issues, non-cooperative bargaining model with uncertainty. Automatica 2008, 44, 1488-1503. [CrossRef]

7. Madani, K. Game theory and water resources. J. Hydrol. 2010, 381, 225-238. [CrossRef]

8. Kerachian, R.; Fallahnia, M.; Bazargan-Lari, M.R.; Mansoori, A.; Sedghi, H. A fuzzy game theoretic approach for groundwater resources management: Application of Rubinstein Bargaining Theory. Resour. Conserv. Recycl. 2010, 54, 673-682. [CrossRef]

9. Wei, S.; Yang, H.; Abbaspour, K.; Mousavi, J.; Gnauck, A. Game theory based models to analyze water conflicts in the Middle Route of the South-to-North Water Transfer Project in China. Water Res. 2010, 44, 2499-2516. [CrossRef]

10. Safari, N.; Zarghami, M.; Szidarovszky, F. Nash bargaining and leader-follower models in water allocation: Application to the zarrinehrud river basin, Iran. Appl. Math. Model. 2014, 38, 1959-1968. [CrossRef]

11. Dinar, A.; Hogarth, M. Game theory and water resources: Critical review of its contributions, progress and remaining challenges. Found. Trends Microecon. 2015, 11, 1-139. [CrossRef]

12. Degefu, D.M.; He, W. Water bankruptcy in the mighty Nile river basin. Sustain. Water Resour. Manag. 2016, 2, 29-37. [CrossRef]

13. He, Y.; Yang, J.; Chen, X. Allocating river water in a cooperative way: A case study of the Dongjiang River Basin, South China. Stoch. Environ. Res. Risk Assess. 2018, 32, 3083-3097. [CrossRef]

14. Khachaturyan, M.; Schoengold, K. Applying Interconnected Game Theory to Analyze Transboundary Waters: A Case Study of the Kura-Araks Basin. Water Econ. Policy 2019, 5, 1-32. [CrossRef]

15. Yu, Y.; Tang, P.; Zhao, J.; Liu, B.; Mclaughlin, D. Evolutionary Cooperation in Transboundary River Basins. Water Resour. Res. 2019, 55, 9977-9994. [CrossRef]

16. Rao, Z.; Debski, D.; Webb, D.; Harpin, R. Genetic algorithm-based optimization of water resources allocation under drought conditions. Water Supply. 2010, 10, 517-525. [CrossRef]

17. Reed, P.M.; Hadka, D.; Herman, J.D.; Kasprzyk, J.R.; Kollat, J.B. Evolutionary multiobjective optimization in water resources: The past, present, and future. Adv. Water Resour. 2013, 51, 438-456. [CrossRef]

18. Tabari, M.M.R.; Soltani, J. Multi-Objective Optimal Model for Conjunctive Use Management Using SGAs and NSGA-II Models. Water Resour. Manag. 2013, 27, 37-53. [CrossRef]

19. Sepahvand, R.; Safavi, H.R.; Rezaei, F. Multi-Objective Planning for Conjunctive Use of Surface and Ground Water Resources Using Genetic Programming. Water Resour. Manag. 2019, 33, 2123-2137. [CrossRef]

20. Qi, S.; Wan, L.; Fu, B. Multisource and multiuser water resources allocation based on genetic algorithm. J. Supercomput. 2020, 76, 3222-3230. [CrossRef] 
21. Fu, J.; Zhong, P.; Zhu, F.; Chen, J.; Wu, Y.; Xu, B. Water Resources Allocation in Transboundary River Based on Asymmetric Nash-Harsanyi Leader-Follower Game Model. Water 2018, 10, 270. [CrossRef]

22. Tian, Y.; Cheng, R.; Zhang, X.Y.; Cheng, F.; Jin, Y.C. An Indicator Based Multi-objective Evolutionary Algorithm with Reference Point Adaptation for Better Versatility. IEEE Trans. Evol. Comput. 2017, 22, 609-622. [CrossRef]

23. Griffith, R.E.; Stewart, R.A. A Nonlinear programming technique for the optimization of continuous process systems. Manag. Sci. 1961, 7, 379-392. [CrossRef]

24. Deb, K.; Pratap, A.; Agarwal, S.; Meyarivan, T. A fast and elitist multiobjective genetic algorithm: NSGA-II. IEEE Trans. Evol. Comput. 2002, 6, 182-197. [CrossRef]

25. Yu, X.; Sreekanth, J.; Cui, T.; Pickett, T.; Xin, P. Adaptative DNN emulator-enabled multi-objective optimization to manage aquifer-sea flux interactions in a regional coastal aquifer. Agric. Water Manag. 2021, 245, 106571. [CrossRef]

26. Tian, Y.; Cheng, R.; Zhang, X.; Jin, Y. PlatEMO: A MATLAB Platform for Evolutionary Multi-Objective Optimization. IEEE Comput. Intell. Mag. 2017, 12, 73-87. [CrossRef] 